\title{
An Intersectional Study of LncRNAs and mRNAs Reveals the Potential Therapeutic Targets of Buyang Huanwu Decoction in Experimental Intracerebral Hemorrhage
}

\author{
Hanjin Cuia Tao Liu a,b Pengfei Lia Ali Yangc,d Huajun Zhoue \\ Jiekun $\mathrm{Luo}^{\mathrm{a}}$ En Hu $\mathrm{Hu}^{\mathrm{a}}$ Wang Hu${ }^{\mathrm{a}}$ Yang Wang ${ }^{\mathrm{a}}$ Tao Tang ${ }^{\mathrm{a}}$
}

anstitute of Integrative Medicine, Xiangya Hospital, Central South University, Changsha, bDepartment of Gerontology, Traditional Chinese Medicine Hospital Affiliated to Xinjiang Medical University, Urumqi, 'Institute of Neurology, Xiangya Hospital, Central South University, Changsha, dDepartment of Neurology, Henan Province People's Hospital, Zhengzhou, eInstitute of Neurology, The First College of Clinical Medical Sciences, Three Gorges University, Yichang, China

\section{Key Words}

Intracerebral hemorrhage • Long non-coding RNA (IncRNA) • Messenger RNA (mRNA) • Expression profile $\cdot$ Intersectional study

\begin{abstract}
Background/Aims: Both experimental and clinical studies have revealed satisfactory effects of the traditional Chinese formula Buyang Huanwu decoction (BYHWD) in improving post-intracerebral hemorrhage $(\mathrm{ICH})$ neurological deficiencies. However, the multifaceted mechanisms of BYHWD in ICH treatment are not comprehensively understood. The present study explored various therapeutic targets of BYHWD by using IncRNA and mRNA transcriptomics. Methods: LncRNA and mRNA microarrays were used to identify differentially expressed genes. ICH-induced upregulated genes (ICH vs sham) and BYHWD-induced downregulated genes (BYHWD vs ICH) were first identified. The intersection between these 2 sets was determined to identify $\mathrm{ICH}$-induced highly expressed genes that were reversed by BYHWD. Then, the genes downregulated after ICH and the genes upregulated after BYHWD treatment were used to generate another set of intersections. Gene Ontology and Kyoto Encyclopedia of Genes and Genomes pathway analyses were subsequently performed to determine relative biological functions and signaling transduction pathways according to genes within the intersections. Quantitative real-time PCR was used to validate changes in gene expression observed using the microarray. Finally, a IncRNA-mRNA co-expression network was established to identify links among the genes within the intersections. Results: A total of 18 differentially expressed IncRNAs and 33 differentially expressed mRNAs were identified using 2 IncRNA arrays (ICH vs sham and BYHWD vs ICH). The altered genes were enriched in the hemoglobin complex, oxygen transport and oxygen transporter and were closely


associated with pyruvate metabolism. The co-expression network consisted of 53 nodes and 595 connections (308 positive interactions and 287 negative interactions). Conclusion: The hemoglobin complex, oxygen transport, oxygen transporter activity and pyruvate metabolism are possible therapeutic targets of BYHWD in ICH treatment. The present study provides the basis and direction for future investigations to explore the mechanisms by which BYHWD protects against long-term neurological deficiencies after ICH.

(C) 2018 The Author(s)

Published by S. Karger AG, Basel

\section{Introduction}

Intracerebral hemorrhage (ICH) remains the most lethal and least treatable subtype of stroke worldwide, resulting in a shocking 40\% 30-day mortality and $80 \%$ disability rate [1$3]$. However, advancements in medical and surgical interventions in the last 10 years have failed to produce dramatic improvements in outcomes after ICH, resulting in an unbearable burden [4-6]. Thus, milestones in ICH therapy, particularly for post-ICH neurological deficiencies, are eagerly anticipated.

Traditional Chinese medicine (TCM) has been employed to treat stroke in China for thousands of years [7]. TCM specifically shows satisfactory effectiveness for stroke survivors suffering from long-term motor dysfunctions, swallowing difficulties, cognitive disorders and dysarthria, which are difficult to resolve using Western medicine $[8,9]$. Thus, TCM has been considered an important therapeutic alternative to Western medicine for treating stroke since the last century $[10,11]$. TCM formula is a holistic system, and specific treatments are usually composed of several medicines containing hundreds of components. These components are mixed together to interact with one other to affect an unknown number of cellular targets, concurrently generating systematic effects [12, 13]. The multi-targeted effects of TCM have aroused growing interest in exploring the related mechanisms.

Buyang Huanwu decoction (BYHWD) is the most frequently prescribed formula for stroke treatment (40.32\%) [14]. Evidence-based investigations have found that BYHWD improves neurological deficiencies in both experimental studies and clinical trials [15-17]. Unfortunately, present reports tend to focus on a single target or pathway, and none of these studies has been able to reveal multifaceted regulatory mechanisms of BYHWD after ICH.

Approximately $\quad 99 \%$ of mammalian RNA transcripts are noncoding RNAs (ncRNAs), and the majority of these are long noncoding RNAs (lncRNAs, > 200 nucleotides) [18]. LncRNAs are generally not translated into proteins but actively participate in many essential biological processes by controlling gene expression at the epigenetic, transcriptional and post-transcriptional levels [19]. Therefore, investigations from the perspective of interactions between IncRNAs and mRNAs may capture a more comprehensive regulatory

Fig. 1. Flowchart of the study. Differentially expressed lncRNAs and mRNAs from 2 intersections (ICH vs Sham and BYHWD vs ICH). GO and KEGG analyses were used to explore closely related biological roles and signaling pathways. qRT-PCR was used to validate the changes in genes based on the IncRNA microarrays. The co-expression network was constructed to explore potential interactions between genes.

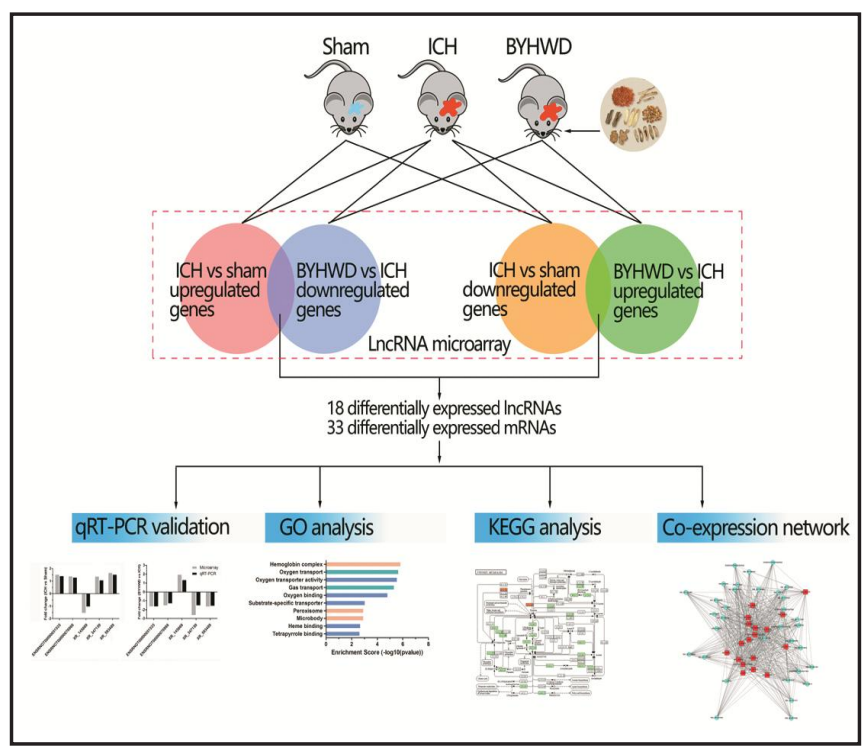


network. Previous studies have found that calycosin (an important active component of BYHWD) exhibits cell proliferation-inhibiting and apoptosis-inducing effects through lncRNA HOTAIR [20]. Another active component of BYHWD, quercetin, is found to be involved in apoptosis promotion via the upregulation of lncRNA MALAT1 to inhibit PI3K/ AKT signaling [21]. However, these single-compound studies are insufficient to reveal the neuroprotective mechanisms of BYHWD in ICH.

Transcriptomics is a powerful tool for detecting global alterations in RNA expression and, consequently, changes in the corresponding proteins [13,22]. A transcriptomics study of Radix Astragali (RA) and Radix Angelicae Sinensis (RAS) (monarch and ministerial drugs of BYHWD) for treating osteoporosis found that the genes galectin-9, CCL-2, CCL-7 and CCL-8 are specifically regulated by these drugs [23]. Another transcriptomics study on a TCM formula containing RA and RAS detected 483 treatment-related genes in 63 functional categories and 25 cancer-related pathways. In addition, the most closely related three pathways are apoptosis, cell cycle regulation and the mitogen-activated protein kinase (MAPK) cascade [24]. Therefore, it is rational to believe that transcriptomics analysis of IncRNAs and mRNAs may help shed light on multifaceted mechanisms of TCM formulas by identifying exact therapeutic targets and their interactions [13].

In the present study, ICH-induced upregulated genes (ICH vs sham) and BYHWDinduced downregulated genes (BYHWD vs ICH) were first identified. Then, the intersection between these 2 sets was determined to identify aberrantly highly expressed genes that were reversed by BYHWD. In addition, the genes downregulated after ICH and the genes upregulated after BYHWD treatment were used to generate another set of intersections. Then, the related biological functions and pathways of the differentially expressed genes in the intersections were determined by Gene Ontology (GO) and Kyoto Encyclopedia of Genes and Genomes (KEGG) pathway analyses. Finally, the lncRNA-mRNA co-expression network within the intersections was analyzed (Fig. 1). In this way, the post-ICH BYHWD-generated genetic changes and their related biological functions and signaling pathways were investigated. These findings will provide a more comprehensive perspective and profound understanding of the mechanism of BYHWD against long-term neurological deficiencies after ICH. Moreover, this study provides a relatively accurate direction for further investigations to identify definite mechanisms.

\section{Materials and Methods}

\section{Animal Preparation}

The Sprague-Dawley (SD) rats used in this study were 8 to 9 weeks of age (male, $180 \mathrm{~g}-220 \mathrm{~g}$ ). The SD rats were cared for in accordance with the Guide for the Care and Use of Laboratory Animals from the National Institutes of Health (NIH Publication No. 85-23, revised 1996). The Committee on the Use and Care of Animals of Central South University (CSU) (201403164) approved the animal protocols. The rats were given free access to water and food and were housed at a room temperature of $25{ }^{\circ} \mathrm{C}$ on a $12 \mathrm{~h}$ light-dark cycle.

\section{BYHWD preparation}

The original composition of BYHWD from the "TCM Prescriptions Dictionary" is listed in Table 1. Dried crude herbs were obtained from the Chinese Medicinal Pharmacy of Xiangya Hospital, Changsha, China. Voucher specimens were deposited at the Laboratory of Institute of Integrative Medicine, CSU. The decoction was prepared and subjected to quality control as previously described. Before intragastric administration, the powder was dissolved in distilled water at a concentration of $(0.13 \mathrm{~g} / \mathrm{ml})$.

\section{ICH induction and animal groups}

Animals were randomly assigned to the sham-operated group (short for the sham + vehicle group), ICH group (short for the ICH + vehicle group) and BYHWD group (short for the ICH + BYHWD-treated group) $(n=3$, per group) (for the randomization of animals, refer to Cheng $\mathrm{T}$ [25]). The rats were fixed in a prone 
Table 1. Components of the Buyang Huanwu decoction. In the order listed in, herbs were combined in a 60:6:4.5:3:3:3 ratio (dry weight)

\begin{tabular}{|c|c|c|c|c|c|}
\hline Latin name & Family & English name & Chinese name & Part used & Batch number \\
\hline Radix astragali & Leguminosae & Astragalus mongholicus & Huang qi & Root & 091201 \\
\hline Radix paeoniae Rubra & Ranunculaceae & Red peony root & Chi shao & Root & 091214 \\
\hline Radix angelicae Sinensis & Umbelliferae & Angelica root & Dang gui & Root & 091206 \\
\hline Rhizoma Ligustici Chuan xiong & Umbelliferae & Sichuan lovage & rhizome Chuanxiong & Root & 100304 \\
\hline Flos Carthami & Feverfew & Carthamus tinctorius & Hong hua & Flower & 100329 \\
\hline Semen persicae & Rosaceae & Peach seed & Tao ren & Seed & 091218 \\
\hline Pheretima Aspergillum & Megascolecidae & Earthworm (Lumbricus) & Di long & Whole animal & 091222 \\
\hline
\end{tabular}

Table 2. Physiological variables of each experimental group (mean \pm SD). Hct: hematocrit; values are expressed as the mean \pm SD

\begin{tabular}{lccccc}
\hline & $\mathrm{pH}$ & $\mathrm{pO}_{2}(\mathrm{mmHg})$ & $\mathrm{pCO}_{2}(\mathrm{mmHg})$ & Hct $(\%)$ & Glucose $(\mathrm{mg} / \mathrm{dL})$ \\
\hline Sham & $7.40 \pm 0.05$ & $79.7 \pm 4.2$ & $44.3 \pm 5.5$ & $45.3 \pm 6.7$ & $98.6 \pm 6.5$ \\
ICH & $7.43 \pm 0.02$ & $81.7 \pm 5.9$ & $42.7 \pm 3.2$ & $45.6 \pm 4.6$ & $104.6 \pm 5.4$ \\
ICH+BYHWD & $7.42 \pm 0.04$ & $83.0 \pm 7.1$ & $42.3 \pm 4.7$ & $43.0 \pm 5.3$ & $107.3 \pm 10.8$ \\
\hline
\end{tabular}

position on a stereotactic instrument (Stoelting Co., Chicago, IL, USA) after being deeply anesthetized with pentobarbital ( $65 \mathrm{mg} / \mathrm{kg}$ i.p.). Then, $2.5 \mu \mathrm{l}$ of $0.5 \mathrm{U}$ collagenase (type VII) in $0.9 \%$ sterile saline was slowly injected into the right globus pallidus (site: $1.4 \mathrm{~mm}$ posterior and $3.2 \mathrm{~mm}$ lateral to the bregma and 5.6 $\mathrm{mm}$ ventral to the cortical surface) at an even speed within $2 \mathrm{~min}$ by using a 26-gauge needle. After the infusion, the needle was kept in place for $5 \mathrm{~min}$. For the sham-operated group, $2.5 \mu \mathrm{l}$ of $0.9 \%$ sterile saline without type VII collagenase was infused into the same site in the same manner [26, 27]. For the ICH + BYHWD-treated group, intragastric administration of BYHWD $(2.05 \mathrm{~g} / \mathrm{kg})$ was performed once a day for 21 days. The $4.36 \mathrm{~g} / \mathrm{kg}$ dose of BYHWD was shown to be effective for mice in our previous study [28], and it was calculated at $2.05 \mathrm{~g} / \mathrm{kg}$ for rats according to surface area conversion. In addition, this dose was confirmed to be effective by preliminary motor functional analysis in animals with ICH (unpublished data). Equal volumes of distilled water were administered to the sham and ICH groups once daily for 21 days. During the whole process, blood glucose, $\mathrm{pH}, \mathrm{PaO}_{2}, \mathrm{PaCO}_{2}$, arterial blood pressure, and hematocrit levels in the animals were monitored (Table 2). Feedback-controlled heating pads were used to maintain the rectal temperature of animals at approximately $37.5^{\circ} \mathrm{C}$. Animals were sacrificed 21 days after ICH induction. Brain tissues around hematoma were obtained for subsequent analysis.

\section{LncRNA and mRNA microarray}

Total RNA was extracted from the right globus pallidus and purified by using an RNeasy Mini Kit (Qiagen, Redwood City, CA, USA). Arraystar Rat lncRNA microarrays (v2.0, containing 13611 lncRNAs and 24626 coding transcripts) were used to detect the expression of lncRNAs and mRNAs in a total of 9 rats (3 groups with 3 replicates). Tissue preparation and microarray hybridization were performed by using an Agilent Gene Expression Hybridization Kit (Agilent Technology, CA, USA). After washing, the arrays were scanned by an Agilent Microarray Scanner and finally analyzed by Agilent Feature Extraction software (version 10.5.1.1). Differentially expressed transcripts (thresholds of $\geq 1$. 3 -fold and $p$ values of $<0.05$ ) were identified by comparing the ICH and sham control groups (ICH vs sham) and the ICH + BYHWD-treated and ICH groups (BYHWD vs ICH). Then, we determined the intersection between the upregulated transcripts in the ICH vs sham groups and the downregulated transcripts in the BYHWD vs ICH groups as well as the intersection between the downregulated transcripts in the ICH vs sham groups and the upregulated transcripts in the BYHWD vs ICH groups in order to identify the mechanism by which BYHWD reverses pathophysiological changes in ICH.

\section{GO and KEGG pathway analyses}

GO (http://www.geneontology.org) analysis was performed to determine the biological roles based on the molecular functions, biological processes, and cellular components of the aberrantly expressed mRNAs; $\mathrm{p}<0.05$ and FDR $<0.05$ were used as thresholds to define markedly enriched GO terms/pathways. Pathway 
analysis (based on KEGG, http://www. genome.jp/kegg/) was performed to explore the pathways significantly enriched in differentially expressed genes.

Quantitative real-time PCR

validation

The portion of the samples remaining after IncRNA microarray was used for quantitative real-time polymerase chain reaction (qRT-PCR) validation. The method was described in Li Q and Wang J [29]. SuperScript III Reverse Transcriptase (Invitrogen, Grand Island, NY, USA) was used to reverse transcribe total RNA into cDNA according to the instructions of the manufacturer. Quantitative real-time (qRT)-PCR (Arraystar) was performed by using the Applied Biosystems ViiA 7 RT-PCR System and $2 \times$ PCR Master Mix. The reaction conditions were as follows: incubation at $95{ }^{\circ} \mathrm{C}$ for $10 \mathrm{~min}$, followed by 40 cycles of $95{ }^{\circ} \mathrm{C}$ for $10 \mathrm{~s}$ and $60{ }^{\circ} \mathrm{C}$ for $1 \mathrm{~min}$. The relative expression levels of lncRNAs were calculated by using the $2^{-\Delta \Delta \mathrm{Ct}}$ method and were normalized by $\beta$-actin levels (R). The primers for each gene are listed in Table 3.

\section{LncRNA and mRNA co-expression network}

A co-expression network (CNC network) was constructed with 18 lncRNAs and 33 mRNAs from the intersections based on correlation analysis. The network was constructed by using Cytoscape software (version 2.8.3, The Cytoscape Consortium, San Diego, CA, USA). The 18 IncRNAs were represented by red squares, and 33 mRNAs were represented by blue nodes. Positive and negative relationships were represented by solid lines and dashed lines, respectively.

\section{Statistics}

SPSS (version 13.0; SPSS Inc., Chicago, IL, USA) was used for statistical analysis. The results were expressed as the mean \pm SEM. Differences between the "sham-operated group" and the "ICH group" and between the "BYHWD group" and the "ICH group" were analyzed by using Student's t-tests. Spearman's correlation analysis was used to detect the relationship between lncRNAs and mRNAs. A p value of $<0.05$ was regarded as statistically significant.

\section{Results}

Identification of mutually expressed IncRNAs and mRNAs among the three groups

In total, 18 differentially expressed lncRNAs were identified from 2 lncRNA arrays (ICH vs sham and BYHWD vs ICH, 3 rats in each group) with a fold-change cut-off of $1.3(\mathrm{p}<0.05)$. Among them, 10 common lncRNAs were identified from the intersection of 131 upregulated lncRNAs (ICH vs sham) and 164 downregulated lncRNAs (BYHWD vs ICH) (Fig. 2A, Table 4). Another 8 common IncRNAs were identified from the intersection of 494 downregulated lncRNAs (ICH vs sham) and 133 upregulated lncRNAs (BYHWD vs ICH) (Fig. 2A, Table 4).

Additionally, 33 differentially expressed mRNAs were identified from 2 lncRNA arrays (ICH vs sham and BYHWD vs ICH, 3 rats in each group) with a fold-change cut-off of 1.3 (p $<0.05$ ). The intersection of 367 upregulated mRNAs (ICH vs sham) and 175 downregulated mRNAs (BYHWD vs ICH) led to the identification of 22 common mRNAs (Fig. 2B, Table 5). The intersection of 459 downregulated mRNAs (ICH vs sham) and 260 upregulated mRNAs (BYHWD vs ICH) led to the identification of 11 common mRNAs (Fig. 2 B, Table 5).

\section{KARGER}




\section{Cellular Physiology Cell Physiol Biochem 2018;46:2173-2186 \begin{tabular}{ll|l} 
and Biochemistry Published online: May 04, 2018 & $\begin{array}{l}\text { (c) } 2018 \text { The Author(s). Published by S. Karger AG, Basel } \\
\text { www.karger.com/cpb }\end{array}$
\end{tabular}

Fig. 2. Hierarchical cluster heat map showing differentially expressed IncRNAs (A) and mRNAs (B) from 2 intersections (ICH vs Sham and BYHWD vs ICH). Each of the three adjacent columns represents samples from the sham-operated group, ICH group and BYHWD group in order. Each row represents genes with IncRNAs (A) and mRNAs (B) labeled in the rightmost column. A bar representing expression level is shown in the upper right-most corner. Red indicates relatively high expression; green indicates relatively low expression; and black indicates no change.

\section{GO and KEGG pathway analysis}

In total, 33 mutually expressed mRNAs were subjected to GO analyses (http://www. geneontology.org). The results showed that the hemoglobin complex (ontology: cellular component, GO: 0005833), oxygen transport (ontology: biological process, GO: 0015671) and oxygen transporter activity (ontology: molecular function, GO: 0005344) were the top 3 GO terms (Fig. 3). In addition, KEGG pathway analysis identified pyruvate metabolism (pathway ID: rno00620) (Fig. 4).

\section{Validation of the Microarray Data Using qRT-PCR}

Five differentially expressed lncRNAs (ENSRNOT00000051535, ENSRNOT00000076988, XR_145869, XR_347159 and XR_593489) were randomly selected for analysis using RT-qPCR to validate the expression changes in lncRNAs detected by RNA-seq. The results of the two methods revealed similar expression patterns of the lncRNAs. Both RNA-seq and RT-qPCR showed that the expression of ENSRNOT00000051535, ENSRNOT00000076988, XR_347159 and XR_593489 was upregulated after ICH but downregulated after BYHWD treatment and that XR_145869 expression decreased after ICH but increased after BYHWD treatment (Fig. 5). RT-qPCR validation indicated good reliability and reproducibility of the IncRNA changes determined by RNA-seq.

\section{LncRNA-mRNA co-expression network}

We constructed a lncRNA and mRNA co-expression network based on the 18 differentially expressed lncRNAs and 33 interacting mRNAs. There were 53 nodes and 595 connections (308 positive interactions

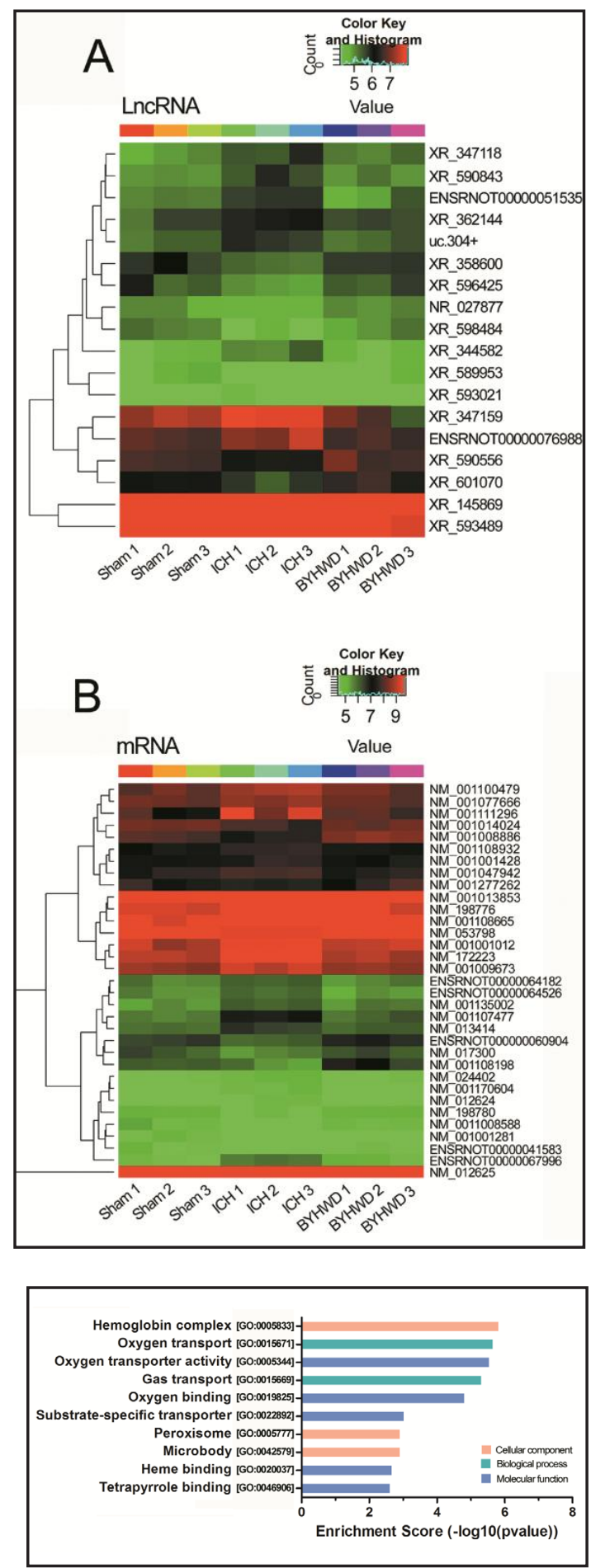

Fig. 3. Gene ontology of differentially expressed mRNAs. The top 10 GO terms associated with the differentially expressed mRNAs. The differentially expressed mRNAs for GO analysis were obtained by comparing the sham, ICH and BYHWD groups. 


\section{Cellular Physiology Cell Physiol Biochem 2018;46:2173-2186 \begin{tabular}{l|l} 
and Biochemistry $10.1159 / 000489547$ \\
Published online: May 04, 2018 & $\begin{array}{l}\text { O 2018 The Author(s). Published by S. Karger AG, Basel } \\
\text { www.karger.com/cpb }\end{array}$ \\
\cline { 2 - 3 } &
\end{tabular}}

Cui et al.: An Intersectional Study of LncRNAs and MRNAs in ICH

Table 4. 18 differentially expressed IncRNAs identified from 2 IncRNA arrays (ICH vs sham, BYHWD vs ICH). Seq name: sequence name. $P$ value: $P$ value calculated by using unpaired $t$-tests. Fold change: the absolute ratio (no log scale) of the normalized intensities between two groups (ICH vs sham; BYHWD vs ICH). Chr: chromosome number from which the IncRNA is transcribed. Str: the strand of chromosome from which the IncRNA is transcribed; "+" represents the sense strand of the chromosome, and "-" represents the antisense strand of the chromosome. Relationship: intergenic, there are no coding transcripts within $30 \mathrm{~kb}$ of the IncRNA; intronic antisense, RNA molecules that are transcribed from the antisense strand without sharing overlapping exons; and natural antisense, RNA molecules transcribed from the antisense strand and overlapping in part with well-defined spliced sense or intronless sense RNAs. Sham 1 to 3, ICH 1 to 3 and BYHWD 1 to 3 : normalized intensity of each sample ( $\log 2$ transformed)

\begin{tabular}{|c|c|c|c|c|c|c|c|c|c|c|c|c|c|c|c|c|c|c|}
\hline \multirow{2}{*}{ Seq name } & \multicolumn{2}{|c|}{ Pvalue } & \multicolumn{2}{|c|}{ Folt change } & \multicolumn{2}{|c|}{ Relation } & \multirow{2}{*}{ Chr } & \multirow{2}{*}{ str } & \multirow{2}{*}{ Relationship } & \multirow{2}{*}{ Sham 1} & \multirow[b]{2}{*}{ Sham 2} & \multirow[b]{2}{*}{ Sham 3} & \multirow{2}{*}{ ICH 1} & \multirow[b]{2}{*}{ ICH 2} & \multirow[b]{2}{*}{$\mathrm{ICH} 3$} & \multirow[b]{2}{*}{ BY HWD1 } & \multirow[b]{2}{*}{ BYHWD2 } & \multirow[b]{2}{*}{ BYHWD 3} \\
\hline & ICH vs Sham & BYHWD vs ICH & ICH vs Sham & BYHWD vs ICH & ICH vs Sham & BYHWD vs ICH & & & & & & & & & & & & \\
\hline XR_593021 & 0.030 & 0.013 & 1.378 & 1.338 & up & down & Chr 6 & + & intergenic & 4.01 & 3.58 & 3.84 & 4.39 & 4.25 & 4,17 & 3.83 & 3.74 & 3.99 \\
\hline ENSRNOTO00000051535 & 0.0002 & 0.039 & 1.541 & 1.662 & up & down & Chr14 & & intergenic & 5.19 & 5.13 & 5.06 & 5.87 & 5.79 & 5.78 & 4.69 & 4.87 & 5.49 \\
\hline uc.304+ & 0.012 & 0.024 & 1.390 & 1.351 & up & down & Chr1 & + & intergenic & 5.14 & 5.41 & 5.40 & 5.90 & 5.77 & 5.70 & 5.21 & 5.30 & 5.56 \\
\hline XR_.347118 & 0.011 & 0.045 & 1.64 & 1.34 & up & down & Chr 6 & & intergenic & 4.75 & 4.88 & 5.10 & 5.52 & 5.48 & 5.87 & 5.19 & 5.07 & 5.34 \\
\hline XR_362144 & 0.042 & 0.004 & 1.400 & 1.315 & up & down & Chr20 & + & intergenic & 5.20 & 5.65 & 5.70 & 5.91 & 6.00 & 6.01 & 5.55 & 5.68 & 5.56 \\
\hline XR._344582 & 0.045 & 0.014 & 2.004 & 1.646 & up & down & $\mathrm{Chr} 2$ & & $\begin{array}{l}\text { Intronic } \\
\text { antisense }\end{array}$ & 3.53 & 4.48 & 4.51 & 5.08 & 5.02 & 5.43 & 4.59 & 4.22 & 4.57 \\
\hline XR_.593489 & 0.004 & 0.035 & 1.677 & 1.680 & up & down & Chr7 & + & intergenic & 8.10 & 8.19 & 8.01 & 8.77 & 8.68 & 9.07 & 8.38 & 8.21 & 7.69 \\
\hline XR_590843 & 0.008 & 0.024 & 1.560 & 1.490 & up & down & Chr12 & + & intergenic & 4.94 & 5.03 & 4.97 & 5.44 & 5.87 & 5.56 & 4.95 & 5.25 & 4.95 \\
\hline XR_.347159 & 0.005 & 0.042 & 1.39 & 2.66 & up & down & Chr6 & & intergenic & 7.23 & 7.47 & 7.34 & 7.92 & 7.77 & 7.77 & 7.06 & 6.69 & 5.48 \\
\hline ENSRNOT000000076988 & 0.046 & 0.024 & 1.41 & 1.54 & up & down & Chrx & + & intergenic & 6.88 & 6.74 & 6.65 & 7.13 & 7.05 & 7.57 & 6.58 & 6.78 & 6.53 \\
\hline XR_601070 & 0.023 & 0.006 & 1.41 & 1.80 & dwn & up & Chr5 & + & natural antisense & 6.18 & 6.20 & 6.06 & 5.76 & 5.38 & 5.60 & 6.51 & 6.64 & 6.64 \\
\hline XR_.145869 & 0.019 & 0.016 & 1.56 & 1.99 & dwn & up & Chr3 & & intergenic & 9.72 & 9.82 & 10.01 & 9.47 & 8.97 & 9.20 & 10.33 & 9.80 & ${ }^{10.48}$ \\
\hline XR.358600 & 0.020 & 0.000 & 1.49 & 1.41 & dwn & up & Chr12 & . & intergenic & 5.81 & 6.10 & 5.60 & 5.36 & 5.24 & 5.19 & 5.74 & 5.74 & 5.79 \\
\hline XR_596425 & 0.041 & 0.009 & 1.60 & 1.51 & dwn & up & Chr16 & + & intergenic & 6.02 & 5.28 & 5.48 & 5.02 & 4.90 & 4.82 & 5.45 & 5.35 & 5.73 \\
\hline X__598484 & 0.002 & 0.044 & 2.10 & 1.80 & dwn & up & Chr19 & + & natural antisense & 5.28 & 5.14 & 5.07 & 4.13 & 4.61 & 3.70 & 4.81 & 4.95 & 5.24 \\
\hline NR_.027877 & 0.038 & 0.000 & 1.31 & 1.39 & dwn & up & Chr1 & + & exon sense-vvertapping & 5.09 & 5.10 & 4.71 & 4.60 & 4.55 & 4.58 & 5.07 & 5.00 & 5.13 \\
\hline XR_.590556 & 0.007 & 0.024 & 1.39 & 1.51 & dwn & up & Chr1 & & intergenic & 6.72 & 6.64 & 6.60 & 6.22 & 6.31 & 6.02 & 7.06 & 6.61 & 6.65 \\
\hline XR_589953 & 0.007 & 0.044 & 2.22 & 1.70 & dwn & up & Chr1 & . & intergenic & 4.11 & 4.64 & 4.82 & 3.40 & 3.50 & 3.22 & 3.85 & 3.93 & 4.64 \\
\hline
\end{tabular}

Table 5. 33 differentially expressed mRNAs identified from 2 lncRNA arrays (ICH vs sham, BYHWD vs ICH). Seq name: sequence name. $P$ value: $P$ value calculated by using unpaired $t$-tests. Fold change: the absolute ratio (no log scale) of normalized intensities between two groups (ICH vs sham; BYHWD vs ICH). Chr: chromosome number from which the mRNA is transcribed. Str: the strand of chromosome from which the mRNA is transcribed; "+" represents the sense strand of the chromosome, and "-" represents the antisense strand of the chromosome. Gene symbol: gene name. Sham 1 to 3, ICH 1 to 3 and BYHWD 1 to 3: normalized intensity of each sample (log2 transformed)

\begin{tabular}{|c|c|c|c|c|c|c|c|c|c|c|c|c|c|c|c|c|c|c|}
\hline \multirow[b]{2}{*}{ Seq name } & \multicolumn{2}{|c|}{$\begin{array}{l}\text { Pvalue } \\
\end{array}$} & \multicolumn{2}{|c|}{ Foul change } & \multicolumn{2}{|c|}{ Relation } & \multirow[b]{2}{*}{$\mathrm{Chr}$} & \multirow[b]{2}{*}{ Str } & \multirow[b]{2}{*}{ Gene symbol } & \multirow[b]{2}{*}{ Sham 1} & \multirow[b]{2}{*}{ Sham 2} & \multirow[b]{2}{*}{ Sham 3} & \multirow[b]{2}{*}{ ICH 1} & \multirow[b]{2}{*}{ ICH 2} & \multirow[b]{2}{*}{$\mathrm{ICH}_{3}$} & \multirow[b]{2}{*}{ BYHWD 1} & \multirow[b]{2}{*}{ BYHWD 2} & \multirow[b]{2}{*}{ BYHWD 3} \\
\hline & ICH vs Sham & BYHWD vs ICH & ICH vs Sham & BYHWD vs $1 \mathrm{CH}$ & ICH vs Sham & BYHWD vs ICH & & & & & & & & & & & & \\
\hline ENSRNOT00000064182 & 0.033 & 0.035 & 1.31 & 1.38 & up & down & Chr3 3 & & Slc38a11 & 5.89 & 5.51 & 5.61 & 6.08 & 6.00 & 6.12 & 5.34 & 5.61 & 5.83 \\
\hline NM__0011074777 & $\begin{array}{l}0.000 \\
0.036\end{array}$ & $\begin{array}{l}0.005 \\
0.023\end{array}$ & $\begin{array}{l}2.45 \\
0.44\end{array}$ & $\begin{array}{l}1.95 \\
136\end{array}$ & up & down & $\begin{array}{l}\text { Chr1 } 1 \\
\text { Che16 }\end{array}$ & 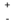 & 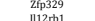 & $\begin{array}{l}5.74 \\
3_{933}-1\end{array}$ & $\begin{array}{l}5.59 \\
438\end{array}$ & 5.76 & 6.94 & 6.88 & $\begin{array}{l}7.15 \\
4.92\end{array}$ & $\begin{array}{c}5.79 \\
4.35\end{array}$ & 5.98 & 6.30 \\
\hline 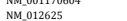 & $\begin{array}{l}0.036 \\
0.006\end{array}$ & $\begin{array}{l}0.003 \\
0.018\end{array}$ & $\begin{array}{l}1.1 .45 \\
1.45\end{array}$ & $\begin{array}{l}1.36 \\
1.46\end{array}$ & 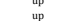 & $\begin{array}{l}\text { down } \\
\text { down }\end{array}$ & $\begin{array}{l}\text { Chr16 } \\
\text { Chr7 }\end{array}$ & : & $\begin{array}{l}\text { III2trit1 } \\
\text { Pmch }\end{array}$ & $\begin{array}{l}3.93 \\
12.95\end{array}$ & $\begin{array}{l}\begin{array}{r}4.38 \\
13.61\end{array} \\
\end{array}$ & 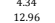 & $\begin{array}{l}\begin{array}{l}4.70 \\
13.70\end{array} \\
\end{array}$ & $\begin{array}{l}\begin{array}{l}4.62 \\
13.43\end{array} \\
\end{array}$ & $\begin{array}{l}4.92 \\
13.56 \\
\end{array}$ & $\begin{array}{l}\begin{array}{l}4.55 \\
13.21\end{array} \\
\text { la }\end{array}$ & $\begin{array}{l}\begin{array}{l}4.13 \\
13.04\end{array} \\
\end{array}$ & $\begin{array}{l}\begin{array}{l}4.42 \\
13.79\end{array} \\
\end{array}$ \\
\hline NM_.013414 & 0.024 & 0.022 & 1.57 & 1.34 & up & down & $\mathrm{Chr} 2$ & & Bgap & 5.88 & 5.89 & 5.81 & 6.68 & 6.48 & 6.37 & 5.95 & 6.16 & 6.16 \\
\hline NM.001108665 & 0.023 & 0.013 & $\begin{array}{l}1.85 \\
1155\end{array}$ & 1.46 & up & down & Chr 5 & 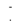 & Mrpl50 & 10.08 & 9.24 & 9.58 & 10.53 & 10.47 & 10.56 & 10.12 & 10.08 & 9.73 \\
\hline $\begin{array}{l}\mathrm{NM} .001001012 \\
\text { NM } 024402\end{array}$ & $\begin{array}{l}0.037 \\
0.022\end{array}$ & $\begin{array}{l}0.016 \\
0.047\end{array}$ & $\begin{array}{l}1.55 \\
1.40\end{array}$ & $\begin{array}{l}1.36 \\
134\end{array}$ & up & $\begin{array}{l}\text { down } \\
\text { downen }\end{array}$ & $\begin{array}{l}\text { Chr } 10 \\
\text { Chrx }\end{array}$ & $:$ & $\begin{array}{l}\text { Oll1 } 1405 \\
\text { Alapp }\end{array}$ & $\begin{array}{l}9.28 \\
4.29\end{array}$ & $\begin{array}{l}8.66 \\
424\end{array}$ & $\begin{array}{l}8.86 \\
455\end{array}$ & $\begin{array}{l}9.48 \\
467\end{array}$ & $\begin{array}{l}9.46 \\
488\end{array}$ & $\begin{array}{l}9.76 \\
498\end{array}$ & $\begin{array}{l}9.05 \\
444\end{array}$ & $\begin{array}{l}9.100 \\
4222\end{array}$ & $\begin{array}{l}9.22 \\
4.64\end{array}$ \\
\hline $\begin{array}{l}\text { NM__202402 } \\
\text { NM_198776 }\end{array}$ & $\begin{array}{l}0.022 \\
0.003\end{array}$ & $\begin{array}{l}0.047 \\
0.025\end{array}$ & $\begin{array}{l}{ }_{2.140} \\
2.29\end{array}$ & $\begin{array}{l}\begin{array}{l}1.34 \\
1.90\end{array} \\
1.90\end{array}$ & $\begin{array}{l}\text { up } \\
\text { up } \\
\text { s. }\end{array}$ & $\begin{array}{l}\text { down } \\
\text { down }\end{array}$ & $\begin{array}{l}\text { Chrix } \\
\text { Chr1 }\end{array}$ & & $\begin{array}{l}\text { Akap4 } \\
\text { Hbb-b1 }\end{array}$ & $\begin{array}{l}4.29 \\
9.29 \\
9\end{array}$ & $\begin{array}{l}4.24 \\
9.30\end{array}$ & $\begin{array}{l}4.55 \\
9.18 \\
9.18\end{array}$ & $\begin{array}{l}4.67 \\
10.64\end{array}$ & $\begin{array}{l}4.88 \\
10.08\end{array}$ & $\begin{array}{l}4.98 \\
10.62\end{array}$ & $\begin{array}{l}4.41 \\
9.69\end{array}$ & $\begin{array}{l}4.22 \\
9.74 \\
\end{array}$ & $\begin{array}{l}4.64 \\
9.13 \\
9\end{array}$ \\
\hline ENSRNOTO00000067996 & 0.000 & 0.010 & 2.26 & 1.77 & up & down & Chr8 & & RGD1560775 & 4.66 & 4.56 & 4.47 & 5.68 & 5.82 & 5.74 & 5.15 & 5.04 & 4.58 \\
\hline NM__001135002 & 0.005 & 0.036 & 1.56 & 1.36 & up & down & Chr 20 & & Clps12 & 5.32 & 5.65 & 5.39 & 6.16 & 5.97 & 6.15 & 5.41 & 5.83 & 5.73 \\
\hline $\begin{array}{l}\text { NM_001001428 } \\
\text { NM 0 00108932 }\end{array}$ & $\begin{array}{l}0.015 \\
0008\end{array}$ & $\begin{array}{l}0.023 \\
0.022\end{array}$ & ${ }_{1.43}^{1.41}$ & $\begin{array}{l}{ }_{1.43}^{1.32} \\
132\end{array}$ & up & $\begin{array}{l}\text { down } \\
\text { down }\end{array}$ & $\begin{array}{l}\mathrm{Cht}_{\mathrm{Ch}} 20 \\
\mathrm{Chr} 20\end{array}$ & : & $\begin{array}{l}\text { Orl1748 } \\
\text { Tynst1 }\end{array}$ & $\begin{array}{l}7.03 \\
7.11\end{array}$ & $\begin{array}{l}7.16 \\
740\end{array}$ & $\begin{array}{l}6.99 \\
727\end{array}$ & $\begin{array}{l}7.34 \\
766\end{array}$ & $\begin{array}{l}7.69 \\
765\end{array}$ & $\begin{array}{l}7.64 \\
7.68\end{array}$ & $\begin{array}{l}7.16 \\
733 \\
733\end{array}$ & $\begin{array}{l}7.10 \\
729\end{array}$ & 6.86 \\
\hline 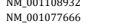 & $\begin{array}{l}0.008 \\
0.013\end{array}$ & $\begin{array}{l}0.002 \\
0.014\end{array}$ & $\begin{array}{l}1.32 \\
1.33\end{array}$ & $\begin{array}{l}1.32 \\
1.37\end{array}$ & $\begin{array}{l}\text { up } \\
\text { up } \\
\text { up }\end{array}$ & $\begin{array}{l}\text { down } \\
\text { down }\end{array}$ & $\begin{array}{l}\text { Chr20 } \\
\text { Chr11 }\end{array}$ & + & $\begin{array}{c}\text { Tysynd1 } \\
\text { RGD1308065 }\end{array}$ & $\begin{array}{l}7.11 \\
8.31 \\
8.31\end{array}$ & $\begin{array}{l}7.40 \\
8.21\end{array}$ & $\begin{array}{l}7.27 \\
8.05\end{array}$ & $\begin{array}{l}7.76 \\
8.59 \\
8.59\end{array}$ & $\begin{array}{l}7.75 \\
8.50\end{array}$ & $\begin{array}{l}7.788 \\
8.72\end{array}$ & $\begin{array}{l}7.33 \\
8.27\end{array}$ & $\begin{array}{l}7.29 \\
8.21\end{array}$ & $\begin{array}{l}7.15 \\
7.97 \\
7.97\end{array}$ \\
\hline NM_001100479 & $\begin{array}{l}0.005 \\
0.005\end{array}$ & $\begin{array}{l}0.017 \\
0.017\end{array}$ & $\begin{array}{l}1.133 \\
1.64\end{array}$ & $\begin{array}{l}1.37 \\
1.49\end{array}$ & up & down & Chr13 & & 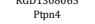 & $\begin{array}{l}8.31 \\
8.00\end{array}$ & $\begin{array}{l}8.21 \\
8.36\end{array}$ & $\begin{array}{l}8.07 \\
8.07 \\
8\end{array}$ & $\begin{array}{l}8.59 \\
8.74\end{array}$ & $\begin{array}{l}8.50 \\
8.87\end{array}$ & $\begin{array}{l}8.97 \\
8.97\end{array}$ & $\begin{array}{l}8.27 \\
8.39\end{array}$ & $\begin{array}{l}8.21 \\
8.43\end{array}$ & 8.02 \\
\hline $\mathrm{NM}_{-} 172223$ & 0.004 & & 1.61 & 1.72 & up & down & $\mathrm{Chr}_{3}$ & & Pxmp4 & 9.04 & 8.98 & 8.87 & 9.58 & 9.53 & 9.86 & 8.88 & 9.02 & 8.72 \\
\hline NM.012624 & $\begin{array}{l}0.021 \\
0.032\end{array}$ & $\begin{array}{l}0.040 \\
0.049\end{array}$ & $\begin{array}{l}1.33 \\
299\end{array}$ & 1.30 & up & down & $\mathrm{Chr}^{\mathrm{Ch} 2}$ & + & $\begin{array}{l}\text { Pldr } \\
\text { Pdr }\end{array}$ & $\begin{array}{l}4.23 \\
8.11\end{array}$ & $\begin{array}{l}4.08 \\
7.07\end{array}$ & 4.23 & $\begin{array}{l}4.39 \\
947\end{array}$ & $\begin{array}{l}4.67 \\
831\end{array}$ & $\begin{array}{l}4.71 \\
9.29 \\
9\end{array}$ & $\begin{array}{l}4.14 \\
807\end{array}$ & $\begin{array}{l}4.11 \\
89313\end{array}$ & 4.37 \\
\hline & $\begin{array}{l}0.032 \\
0.012\end{array}$ & $\begin{array}{l}0.049 \\
0.007\end{array}$ & $\begin{array}{l}2.91 \\
1.44\end{array}$ & $\begin{array}{l}2.08 \\
1.46\end{array}$ & $\begin{array}{l}\text { up } \\
\text { up }\end{array}$ & $\begin{array}{l}\text { down } \\
\text { down }\end{array}$ & $\begin{array}{l}\text { Chr1 } \\
\text { Chr } 9\end{array}$ & & LoC689064 & $\begin{array}{l}8.11 \\
876\end{array}$ & 7.07 & $\begin{array}{l}7.26 \\
850\end{array}$ & $\begin{array}{l}9.47 \\
928\end{array}$ & $\begin{array}{l}8.31 \\
896\end{array}$ & $\begin{array}{l}9.29 \\
9.23\end{array}$ & $\begin{array}{l}8.07 \\
864\end{array}$ & $\begin{array}{l}8.13 \\
8.67\end{array}$ & $\begin{array}{l}7.71 \\
8.52 \\
852\end{array}$ \\
\hline NM_001047943 & $\begin{array}{l}0.012 \\
0.033\end{array}$ & $\begin{array}{l}0.0017 \\
0.017\end{array}$ & 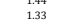 & $\begin{array}{l}1.46 \\
1.37\end{array}$ & $\begin{array}{l}\text { up } \\
\text { up } \\
\text { s. }\end{array}$ & $\begin{array}{l}\text { down } \\
\text { down }\end{array}$ & 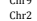 & + & $\begin{array}{l}\text { Fastrka2 } \\
\text { Loc499541 }\end{array}$ & $\begin{array}{l}8.86 \\
7.25\end{array}$ & $\begin{array}{l}8.61 \\
7.61\end{array}$ & $\begin{array}{l}8.50 \\
7.63 \\
\end{array}$ & $\begin{array}{l}9.28 \\
7.96\end{array}$ & $\begin{array}{l}8.96 \\
7.84\end{array}$ & $\begin{array}{l}9.23 \\
7.93\end{array}$ & $\begin{array}{l}8.64 \\
7.26\end{array}$ & $\begin{array}{l}8.67 \\
7.45 \\
7.45\end{array}$ & $\begin{array}{l}8.52 \\
7.64\end{array}$ \\
\hline NM__001013853 & 0.010 & 0.033 & 1.57 & 1.42 & uр & down & Chr10 & & Hba:a 1 & 9.62 & 9.62 & 9.62 & 10.44 & 10.37 & 9.99 & 9.87 & 9.79 & 9.63 \\
\hline ENSRNOTO00000064526 & 0.030 & 0.005 & 1.31 & 1.52 & up & down & Chr12 & & & & 5.43 & 5.48 & 5.99 & 5.86 & 6.02 & 5.18 & 5.53 & 5,34 \\
\hline $\begin{array}{l}\text { NM_.053798 } \\
\text { NM } 0101108\end{array}$ & $\begin{array}{l}0.000 \\
033\end{array}$ & $\begin{array}{l}0.045 \\
0.030\end{array}$ & $\begin{array}{l}1.33 \\
2.242\end{array}$ & $\begin{array}{l}1.47 \\
210\end{array}$ & $\begin{array}{l}\text { down } \\
\text { down }\end{array}$ & up & Chrs & ; & 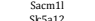 & $\begin{array}{l}9.80 \\
-533\end{array}$ & 9.85 & 9.75 & 9.38 & 9.42 & 9.36 & 10.22 & 10.04 & 9.57 \\
\hline 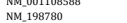 & $\begin{array}{l}0.33 \\
0.012\end{array}$ & $\begin{array}{l}0.030 \\
0.025\end{array}$ & $\begin{array}{l}2.242 \\
1.46\end{array}$ & $\begin{array}{l}2.10 \\
1.37\end{array}$ & $\begin{array}{l}\text { down } \\
\text { down }\end{array}$ & $\begin{array}{l}\text { up } \\
\text { up }\end{array}$ & $\begin{array}{l}\mathrm{Chr}^{\mathrm{Ch} 3} \\
\mathrm{Chr} 3\end{array}$ & : & 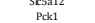 & $\begin{array}{l}5.33 \\
5.17\end{array}$ & $\begin{array}{l}4.61 \\
5.00\end{array}$ & 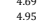 & $\begin{array}{l}4.12 \\
4.43\end{array}$ & $\begin{array}{l}3.67 \\
4.70\end{array}$ & $\begin{array}{l}3.01 \\
4.36 \\
4.36\end{array}$ & $\begin{array}{l}4.76 \\
4.84\end{array}$ & $\begin{array}{l}{ }_{4.91}^{4.91} \\
4.92\end{array}$ & $\begin{array}{l}4.66 \\
5.09\end{array}$ \\
\hline NM_001001281 & 0.017 & 0.046 & 2.06 & 1.66 & down & up & Chr1 & + & Olr75 & 4.53 & 4.85 & 4.58 & 3.88 & 3.81 & 3.10 & 4.25 & 4.44 & 4.30 \\
\hline NM__01277: & 0.023 & & 1.56 & 1.73 & down & up & Chr1 & & Tnnt1 & 7.77 & 7.16 & 7.35 & 6.77 & 6.83 & 6.74 & 7.09 & 7.65 & 7.98 \\
\hline $\begin{array}{l}\text { ENSRNOTO000000041583 } \\
\text { NM 0101108198 }\end{array}$ & $\begin{array}{l}0.037 \\
0041\end{array}$ & $\begin{array}{l}0.044 \\
0.016\end{array}$ & $\begin{array}{l}1.69 \\
164\end{array}$ & $\begin{array}{l}1.47 \\
271\end{array}$ & $\begin{array}{l}\text { down } \\
\text { down }\end{array}$ & up & $\begin{array}{l}\mathrm{Chr} 2 \\
\mathrm{Chr} 9\end{array}$ & & $\begin{array}{l}\text { AABR060201313.1 } \\
\text { Guralb }\end{array}$ & $\begin{array}{l}4.97 \\
6.11\end{array}$ & $\begin{array}{l}4.17 \\
6.05\end{array}$ & $\begin{array}{l}4.52 \\
6.212 \\
\end{array}$ & $\begin{array}{l}3.84 \\
5.99\end{array}$ & 3.63 & $\begin{array}{l}3.91 \\
5.30\end{array}$ & 4.03 & 4.61 & 4.42 \\
\hline 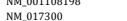 & $\begin{array}{l}0.041 \\
0.026\end{array}$ & $\begin{array}{l}0.016 \\
0.015\end{array}$ & $\begin{array}{l}1.64 \\
1.57\end{array}$ & $\begin{array}{l}2.71 \\
1.62\end{array}$ & $\begin{array}{l}\text { down } \\
\text { down }\end{array}$ & up & $\begin{array}{l}\text { Chr } 9 \\
\text { Chr5 }\end{array}$ & & $\begin{array}{c}\text { Gucalb } \\
\text { Baat }\end{array}$ & $\begin{array}{l}6.11 \\
6.47\end{array}$ & $\begin{array}{l}6.05 \\
6.25 \\
6\end{array}$ & $\begin{array}{l}6.122 \\
5.95\end{array}$ & $\begin{array}{l}5.91 \\
5.36\end{array}$ & $\begin{array}{l}5.44 \\
5.61\end{array}$ & 5.30 & $\begin{array}{l}7.48 \\
6.24\end{array}$ & $\begin{array}{l}7.08 \\
6.50\end{array}$ & $\begin{array}{l}6.41 \\
6.07\end{array}$ \\
\hline NM_.001014024 & $\begin{array}{l}0.019 \\
0.019\end{array}$ & 0.035 & 1.39 & $\begin{array}{l}1.02 \\
1.33 \\
\end{array}$ & down & up & Chr1 & + & $\begin{array}{l}\text { Dadi } \\
\text { Orai3 }\end{array}$ & $\begin{array}{l}8.37 \\
8.32\end{array}$ & 8.22 & 8.30 & 7.91 & 7.93 & 7.56 & $\begin{array}{l}8.22 \\
8.22\end{array}$ & 8.12 & 8.30 \\
\hline NM__01008886 & 0.006 & 0.000 & 1.46 & 2.14 & down & up & Chr20 & & RT1.53 & 8.07 & 7.98 & 7.87 & 7.26 & 7.51 & 7.52 & 8.42 & 8.66 & 8.51 \\
\hline ENSRNOT000000060904 & 0.008 & 0.013 & 1.37 & 1.70 & down & up & $\mathrm{Chr} 2$ & & Ipo11 & 6.36 & 6.48 & 6.58 & 5.89 & 6.02 & 6.14 & 6.78 & 6.90 & 6.67 \\
\hline
\end{tabular}

and 287 negative interactions) in this co-expression network. Among these connections, NM_198780 (Gene symbol PCK1) and XR_598484 (Gene symbol: LOC103694317) had the closest relationship, with a PCC of 0.984 (Fig. 6). 
Fig. 4. KEGG pathway analysis and detailed network of "pyruvate metabolism". KEGG pathway analysis identified pyruvate metabolism as the most correlated signaling pathway. Each green box represents a specific gene or key enzyme. Each white box represents a speciesspecific pathway. Black outlines represent the lack of significant change. The representative significance of each related symbol is shown below. The differentially expressed mRNAs for KEGG pathway analysis were obtained by comparing the sham, ICH and BYHWD groups.

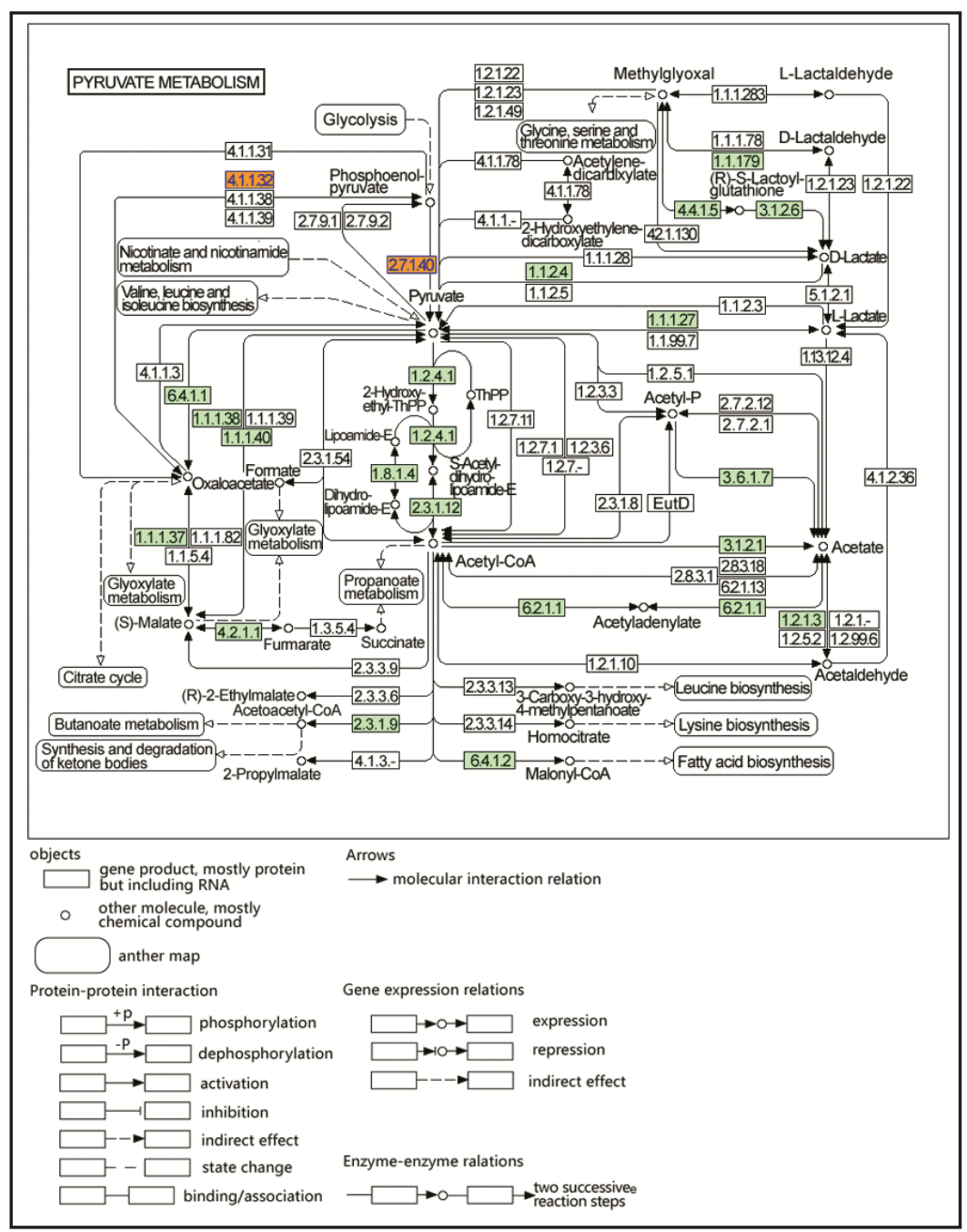

Fig. 5. qRT-PCR validation of 5 randomly selected lncRNAs from the intersection. The results showed that the expression of the lncRNAs ENSRNOT00000051535, ENSRNOT00000076988, XR_347159 and XR_593489 was upregulated after ICH but downregulated after BYHWD treatment, whereas the expression of XR_145869 decreased after ICH but in-

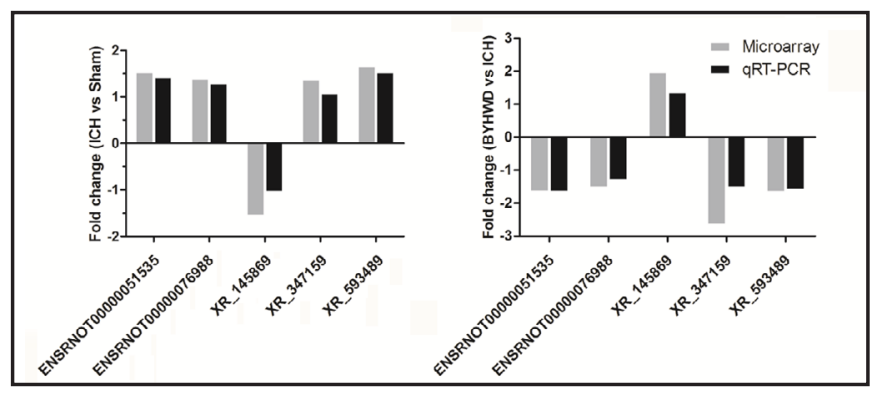
creased after BYHWD treatment. The trends in expression of randomly selected lncRNAs detected by qRT-PCR were similar to those detected by the IncRNA microarray. The remaining portions of the samples from the lncRNA microarray were used for quantitative real-time polymerase chain reaction (qRT-PCR) validation.

\section{Discussion}

This study is the first to identify intersections between IncRNA and mRNA expression profiles in experimental ICH treated with BYHWD. Eighteen differentially expressed lncRNAs and 33 differentially expressed mRNA were identified from 2 lncRNA arrays (ICH vs sham and BYHWD vs ICH). The functions of the altered genes from the intersections were 


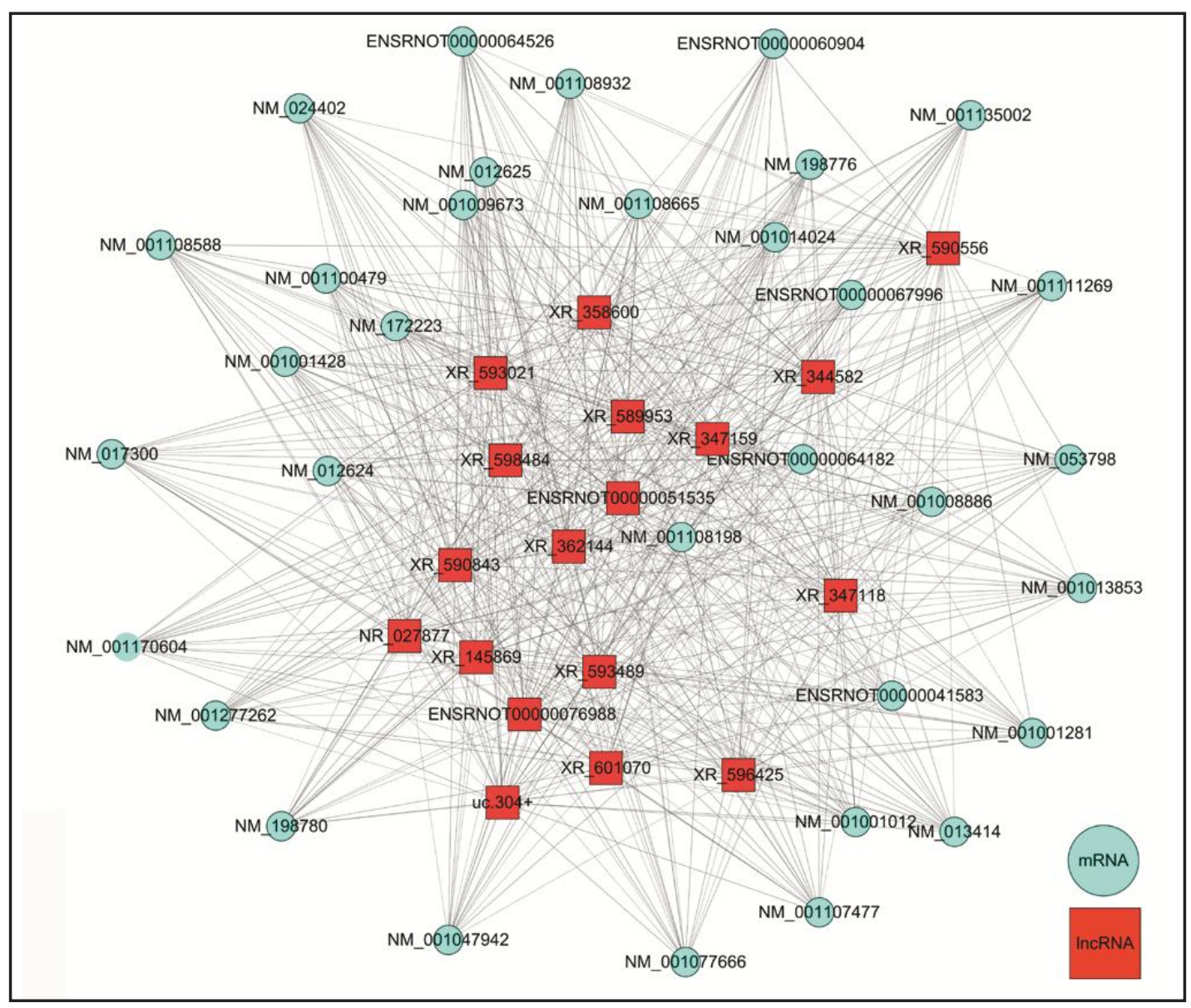

Fig. 6. Co-expression network of IncRNAs and mRNAs from the 2 intersections. Red dots represent lncRNAs, and blue dots represent correlated mRNAs. There were 53 nodes and 595 connections (308 positive interactions and 287 negative interactions) in this co-expression network.

enriched in the hemoglobin complex, oxygen transport and oxygen transporter activity. In addition, these genes were most closely associated with pyruvate metabolism. The genes at the intersections and their related biological functions and pathways might indicate the potential therapeutic targets of BYHWD in ICH treatment.

The hemoglobin complex, oxygen transport and oxygen transporter activity were the top 3 GO terms. All of these molecules and processes involve the same 2 genes, namely, HBA-A1 and HBB-B1. These genes were upregulated after ICH. Hemoglobin (Hb) is a gas transporter with two $\alpha$ chains (hemoglobin alpha [HBA-A1]) and two $\beta$ chains (hemoglobin beta [HBB-B1]). Each chain contains a heme subunit that binds to and delivers oxygen; four oxygen molecules are transported per molecule of hemoglobin [30, 31]. After ICH, high levels of $\mathrm{Hb}$ at lesions usually arise from the following 2 sources: the release of a large fraction of $\mathrm{Hb}$ from the hematoma after red blood cell lysis $[32,33]$ and the expression of the nonerythroid fraction of $\mathrm{Hb}$ in neurons and activated macrophages in response to hypoxia inducible factor-1 (HIF-1 $\alpha$ ) and erythropoietin (EPO) [34-36]. Heme and iron are also found to significantly increase mRNA levels of HBA and HBB in neurons and glial cells of ipsilateral basal ganglia after ICH [37]. In addition, $\mathrm{Hb}$ is a potent cytotoxic chemical [38]. Severe brain edema and high levels of sodium ions are detected $24 \mathrm{~h}$ after cerebral infusion of $\mathrm{Hb}$ [39]. Other studies have found that $\mathrm{Hb}$ and its degradation products, such as iron, carbon monoxide, and biliverdin, participate in oxidative damage and inflammation responses, which result in cell death $[32,40]$. Dead cells further trigger a variety of "danger" signals to activate the infiltration of leukocytes and microglia into the cerebral parenchyma, thereby 
aggravating inflammatory injury [32, 41].

Previous studies have demonstrated the accumulation of lactate, which results in low $\mathrm{pH}$ values in the hematoma and perihematomal regions [42], as also demonstrated by our group (unpublished data). According to the Bohr effect, an acidic environment causes oxygen to dissociate from hemoglobin, leading to increased deoxyhemoglobin and dissociated oxygen. These data support the lack of hypoxia in perihemorrhagic areas [43]. However, excess levels of oxygen usually generate superoxides [44]. When the production of superoxides exceeds the capacity of antioxidant enzymes, oxygen free radicals can inactivate various enzymes, destroy membrane functions, cause DNA damage, and finally result in neuronal death [27, $44,45]$. Hyperoxia exposure in ischemic rats results in significant hippocampal neuronal death and behavioral deficiencies at 7 and 30 days [46]. Hyperoxia is also found to worsen neurological outcomes in a rat model of traumatic brain injury with hemorrhagic shock [47]. Other clinical studies have confirmed that hyperoxia is independently correlated with longterm poor neurological outcomes and in-hospital mortality among patients with brain injury $[48,49]$. Therefore, excessive oxygen is a major factor mediating Hb-induced neurotoxicity.

Increased $\mathrm{Hb}$ levels not only induce oxygen toxicity to kill neurons but also promote the overexpression of nitric oxide synthase (NOS) and nitric oxide (NO) to disrupt the bloodbrain barrier (BBB) [50]. Cerebral Hb injection-enhanced BBB permeability is mediated by increased NOS and NO levels through the regulation of tight junction proteins (zonula occludens-1, claudin-5, occludin, and junctional adhesion molecule-1). In addition, brain edema formation due to BBB leakage is one of the most lethal secondary injuries after ICH and is believed to be a strong predictor of adverse functional outcomes [51]. Moreover, endothelial cytotoxicity can become aggravated when the free radical NO and superoxide exist together and act in a synergistic manner [52]. This may lead to further BBB impairment and more severe cerebral edema. Thus, Hb-induced brain injury is partly mediated by exceedingly high levels of oxygen and NO.

After BYHWD administration, as the levels of HBA-A1 and HBB-B1 decreased, the hemoglobin complex level, oxygen transport and oxygen transporter activity decreased as well, followed by the alleviation of a series of impairments induced by $\mathrm{Hb}$ and peroxidation. Therefore, it is reasonable to hypothesize that BYHWD reverses $\mathrm{Hb}$-induced oxidative damage after ICH by regulating the HBA-A1 and HBB-B1 genes. A series of data has demonstrated antioxidative functions of BYHWD. Pretreatment with BYHWD greatly reduces the level of reactive oxygen species (ROS) in kidneys of rats after brain death [53]. BYHWD also enhances the activity of superoxide dismutase (SOD) in rats with myocardial ischemia [54]. In vitro, BYHWD protects Schwann cells from hydrogen peroxide (H2O2) damage [55]. BYHWD also decrease $\mathrm{H}_{2} \mathrm{O}_{2}$-induced apoptosis in human umbilical vein endothelial cells (HUVECs) [56]. Additionally, pretreatment with BYHWD significantly decreases neuronal NOS activity in the striatum cortex and caudate putamen in rats with permanent focal cerebral ischemia [57]. BYHWD also rescues the myocardium from ischemia by inhibiting the expression of NO and NOS [58]. More importantly, it can be deduced that both oxygen and NO-mediated cytotoxicity result from excess $\mathrm{Hb}$, which is transcribed from the HBA-A1 and HBB-B1 genes. Therefore, in addition to its antioxidative effect, BYHWD is believed to directly control the expression of HBA-A1 and HBB-B1. However, the underlying mechanisms responsible for this effect are still not fully understood. Fortunately, the findings of this study indicate some therapeutic targets of BYHWD and provide interesting directions for future investigations.

KEGG pathway analysis indicated that the genes in the intersection were closely associated with pyruvate metabolism. Phosphoenolpyruvate carboxykinase 1 (PCK1) and pyruvate kinase in liver and red blood cells (PKLR and PK) are involved in this pathway. Our results showed that PCK1 expression decreased after ICH but increased after BYHWD administration, which was opposite to the pattern of PK expression. During the process of pyruvate metabolism, PCK1 catalyzes the generation of phosphoenolpyruvate from oxaloacetate, and PK subsequently catalyzes the transformation of phosphoenolpyruvate to pyruvate. Oxidative stress induced by $\mathrm{Hb}$ exerts a dominant inhibitory effect on the endogenous PCK1 gene [59]. PK is abundantly expressed on red blood cells (RBCs). Thus, 
high levels of PK levels are released from RBCs in hematomas. Although PK is upregulated after ICH, the reduced levels of PCK1 are unable to catalyze the production of sufficient phosphoenolpyruvate, the substrate of PK. As a result, low levels of pyruvate are generated after ICH. Pyruvate can ameliorate ICH-induced brain injury and protect neurons from $\mathrm{Hb}$ induced apoptosis [60]. Therefore, changes in PCK1/PK facilitate neuroprotection following ICH.

Our study found that BYHWD increased the mRNA level of PCK1. This increase might be associated with the reduction in ROS levels by BYHWD, which ameliorates the effects of oxidative stress on PCK1. In addition, CNC network analysis indicated that PCK1 was positively correlated with lncRNA LOC103694317. These findings imply that BYHWD might regulate PCK1 mRNA expression via LOC103694317. However, it is difficult to interpret the decrease in PK after BYHWD treatment based on the present evidence. According to the CNC network, PK mRNA was negatively correlated with XR_358600 (Gene symbol: LOC102546487, PCC: -0.878). It is possible that BYHWD downregulates PK by modulating LOC102546487. In summary, BYHWD might partly rescue the disruptions in pyruvate metabolism by regulating the mRNA expression of the key enzymes PCK1 and PK.

\section{Conclusion}

In conclusion, the present study is the first to demonstrate potential therapeutic targets of BYHWD in ICH treatment at the mRNA and IncRNA levels. These targets are the hemoglobin complex, oxygen transport, oxygen transporter activity and pyruvate metabolism. The findings of the present study provide a basis and direction for future investigations to explore the mechanisms by which BYHWD protects against long-term neurological deficiencies after ICH.

\section{Disclosure Statement}

No conflict of interests exists.

\section{Acknowledgements}

Funding sources: This study was supported by grants from the National Natural Science Foundation of China (Grant No. 81473573, 81173175, 81202625, 81673719 and 81603414) and the Science Foundation for Young Scientists of Xiangya Hospital, Central South University (Grant No. 2016Q09).

YW, TT and HJC designed the experiments. HJC, TL, PFL, ALY, HJZ, EH and WH performed the experiments. YW, TT, HJC and JKL analyzed the data. TL, PFL, ALY, HJZ and JKL provided insights on the interpretation of the results. HJC wrote the manuscript. YW and TT supervised the whole experimental process and the preparation of the manuscript. All authors read and approved the final manuscript.

\section{References}

1 Kim JY, Bae H: Spontaneous intracerebral hemorrhage: Management. J Stroke 2017; 19:28-39.

-2 van Asch CJ, Luitse MJ, Rinkel GLJ, van der Tweel I, Algra A, Klijn CJ: Incidence, case fatality, and functional outcome of intracerebral haemorrhage over time, according to age, sex, and ethnic origin: A systematic review and meta-analysis. Lancet Neurol 2010;9:167-176.

-3 Lan X, Han X, Li Q, Yang QW, Wang J: Modulators of microglial activation and polarization after intracerebral haemorrhage. Nat Rev Neurol 2017;13:420-433. 


\section{Cellular Physiology Cell Physiol Biochem 2018;46:2173-2186 \begin{tabular}{l|l} 
DOI: 10.1159/000489547 & Ond Biochemistry \\
Published online:May 04, 2018 & $\begin{array}{l}\text { 2018 The Author(s). Published by S. Karger AG, Basel } \\
\text { www.karger.com/cpb }\end{array}$
\end{tabular}}

Cui et al.: An Intersectional Study of LncRNAs and MRNAs in ICH

-4 Hemphill JC, Greenberg SM, Anderson CS, Becker K, Bendok BR, Cushman M, Fung GL, Goldstein JN, Macdonald RL, Mitchell PH, Scott PA, Selim MH, Woo D: Guidelines for the management of spontaneous intracerebral hemorrhage. Stroke 2015;46:2032-2060.

5 Kang D: Intracerebral hemorrhage: Large disease burden but less therapeutic progress. J Stroke 2017;19:12.

6 Murray CJL, Lopez AD: Measuring the global burden of disease. New Engl J Med 2013;369:448-457.

7 Chang C, Lee Y, Lin C, Chang C, Chiu C, Chou L, Sun M, Yen H: Characteristics of traditional Chinese medicine usage in patients with stroke in Taiwan: A nationwide population-based study. J Ethnopharmacol 2016;186:311-321.

8 Zhang X, Liu X, Kang D: Traditional chinese patent medicine for acute ischemic stroke. Medicine 2016;95:e2986.

9 Xu S, Pang Q Lin Z, Zhang N: Effect of integrated traditional Chinese and Western medicine therapy for acute hypertensive intracerebral hemorrhage: A meta-analysis. Artif Cells Nanomed Biotechnol 2017;45:16.

10 Yeh M, Chiu W, Wang Y, Lo C: An investigation of the use of traditional chinese medicine and complementary and alternative medicine in stroke patients. Holist Nurs Pract 2017;31:400-407.

11 Gong X, Sucher NJ: Stroke therapy in traditional Chinese medicine (TCM): Prospects for drug discovery and development (Reprinted from Current Trends, vol 20, pg 191-196, 1999). Phytomedicine 2002;9:478-484.

12 Chen H, Qi S, Shen J: One-Compound-Multi-Target: Combination prospect of natural compounds with thrombolytic therapy in acute ischemic stroke. Curr Neuropharmacol 2017;15:134-156.

13 Liu Y, Ai N, Liao J, Fan X: Transcriptomics: A sword to cut the Gordian knot of traditional Chinese medicine. Biomark Med 2015;9:1201-1213.

14 Hung I, Hung Y, Wang L, Hsu S, Chen H, Tseng Y, Kuo C, Hu W, Li T: Chinese herbal products for ischemic stroke. AmJ Chin Med 2015;43:1365-1379.

15 Wei RL, Teng HJ, Yin B, Xu Y, Du Y, He FP, Chu KT, Luo BY, Zheng GQ: A systematic review and meta-analysis of buyang huanwu decoction in animal model of focal cerebral ischemia. Evid Based Complement Alternat Med 2013;2013:138484.

-16 Hao C, Wu F, Shen J, Lu L, Fu D, Liao W, Zheng G: Clinical efficacy and safety of buyang huanwu decoction for acute ischemic stroke: A systematic review and Meta-Analysis of 19 randomized controlled trials. EvidBased Compl Alt 2012;2012:1-10.

-17 Li HQ, Wei JJ, Xia W, Li JH, Liu AJ, Yin SB, Wang C, Song L, Wang Y, Zheng GQ, Fan JP: Promoting blood circulation for removing blood stasis therapy for acute intracerebral hemorrhage: A systematic review and meta-analysis. Acta Pharmacol Sin 2015;36:659-675.

18 Wan P, Su W, Zhuo Y: The role of long noncoding RNAs in neurodegenerative diseases. Mol Neurobiol 2017;54:2012-2021.

19 Zhu J, Fu H, Wu Y, Zheng X: Function of IncRNAs and approaches to IncRNA-protein interactions. Sci China Life Sci 2013;56:876-885.

-20 Chen J, Lin C, Yong W, Ye Y, Huang Z: Calycosin and genistein induce apoptosis by inactivation of HOTAIR/pAkt signaling pathway in human breast cancer MCF-7 cells. Cell Physiol Biochem 2015;35:722-728.

-21 Pan F, Zhu L, Lv H, Pei C: Quercetin promotes the apoptosis of fibroblast-like synoviocytes in rheumatoid arthritis by upregulating IncRNA MALAT1. Int J Mol Med 2016;38:1507-1514.

22 Franks A, Airoldi E, Slavov N: Post-transcriptional regulation across human tissues. Plos Comput Biol 2017;13:e1005535.

23 Choi RC, Gao QT, Cheung AW, Zhu JT, Lau FT, Li J, Li WZ, Chu GK, Duan R, Cheung JK, Ding AW, Zhao KJ, Dong TT, Tsim KW: A chinese herbal decoction, danggui buxue tang, stimulates proliferation, differentiation and gene expression of cultured osteosarcoma cells: Genomic approach to reveal specific gene activation. Evid Based Complement Alternat Med 2011;2011:307548.

24 Zhang P, Wang X, Xiong S, Wen S, Gao S, Wang L, Cao B: Genome wide expression analysis of the effect of the Chinese patent medicine Zilongjin tablet on four human lung carcinoma cell lines. Phytother Res 2011;25:1472-1479.

25 Cheng T, Wang W, Li Q, Han X, Xing J, Qi C, Lan X, Wan J, Potts A, Guan F, Wang J: Cerebroprotection of flavanol (-)-epicatechin after traumatic brain injury via Nrf2-dependent and -independent pathways. Free Radic Biol Med 2016;92:15-28. 


\section{Cellular Physiology Cell Physiol Biochem 2018;46:2173-2186

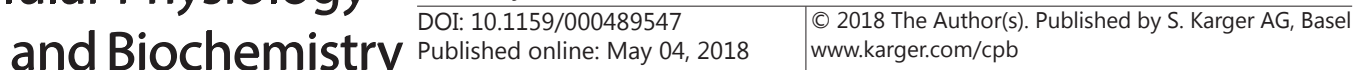

Cui et al.: An Intersectional Study of LncRNAs and MRNAs in ICH

26 Zhou HJ, Zhang HN, Tang T, Zhong JH, Qi Y, Luo JK, Lin Y, Yang QD, Li XQ: Alteration of thrombospondin-1 and -2 in rat brains following experimental intracerebral hemorrhage. Laboratory investigation. J Neurosurg 2010;113:820-825.

-27 Wang M, Hong X, Chang CF, Li Q, Ma B, Zhang H, Xiang S, Heo HY, Zhang Y, Lee DH, Jiang S, Leigh R, Koehler RC, van Zijl PC, Wang J, Zhou J: Simultaneous detection and separation of hyperacute intracerebral hemorrhage and cerebral ischemia using amide proton transfer MRI. Magn Reson Med 2015; doi: 10.1002/ mrm.25690.

-28 Cui H, Yang A, Zhou H, Wang C, Luo J, Lin Y, Zong Y, Tang T: Buyang huanwu decoction promotes angiogenesis via vascular endothelial growth factor receptor-2 activation through the PI3K/Akt pathway in a mouse model of intracerebral hemorrhage. Bmc Complem Altern M 2015;15:91.

29 Li Q Han X, Lan X, Hong X, Li Q, Gao Y, Luo T, Yang Q, Koehler RC, Zhai Y, Zhou J, Wang J: Inhibition of tPAinduced hemorrhagic transformation involves adenosine $\mathrm{A} 2 \mathrm{~b}$ receptor activation after cerebral ischemia. Neurobiol Dis 2017;108:173-182.

-30 Brown HM, Anastasi MR, Frank LA, Kind KL, Richani D, Robker RL, Russell DL, Gilchrist RB, Thompson JG: Hemoglobin: A gas transport molecule that is hormonally regulated in the ovarian follicle in mice and humans1. Biol Reprod 2015;92:26.

-31 Ferrer I, Gomez A, Carmona M, Huesa G, Porta S, Riera-Codina M, Biagioli M, Gustincich S, Aso E: Neuronal hemoglobin is reduced in Alzheimer's disease, argyrophilic grain disease, Parkinson's disease, and dementia with Lewy bodies. J Alzheimers Dis 2011;23:537-550.

-32 Zhou Y, Wang Y, Wang J, Anne Stetler R, Yang Q: Inflammation in intracerebral hemorrhage: From mechanisms to clinical translation. Prog Neurobiol 2014;115:25-44.

-33 Zhang Z, Song Y, Zhang Z, Li D, Zhu H, Liang R, Gu Y, Pang Y, Qi J, Wu H, Wang J: Distinct role of heme oxygenase-1 in early- and late-stage intracerebral hemorrhage in 12-month-old mice. J Cereb Blood Flow Metab 2017;37:25-38.

-34 Altinoz MA, Ince B: Hemoglobins emerging roles in mental disorders. Metabolical, genetical and immunological aspects. Int J Dev Neurosci 2017;61:73-85.

35 Gorr TA, Wichmann D, Pilarsky C, Theurillat JP, Fabrizius A, Laufs T, Bauer T, Koslowski M, Horn S, Burmester T, Hankeln T, Kristiansen G: Old proteins - new locations: Myoglobin, haemoglobin, neuroglobin and cytoglobin in solid tumours and cancer cells. Acta Physiol 2011;202:563-581.

36 Zhou HJ, Tang T, Cui HJ, Yang AL, Luo JK, Lin Y, Yang QD, Li XQ: Thrombin-triggered angiogenesis in rat brains following experimental intracerebral hemorrhage. J Neurosurg 2012;117:920-928.

37 He Y, Hua Y, Keep RF, Liu W, Wang MM, Xi G: Hemoglobin expression in neurons and glia after intracerebral hemorrhage. Acta Neurochir Suppl 2011;111:133-137.

-38 Li Q, Han X, Lan X, Gao Y, Wan J, Durham F, Cheng T, Yang J, Wang Z, Jiang C, Ying M, Koehler RC, Stockwell BR, Wang J: Inhibition of neuronal ferroptosis protects hemorrhagic brain. JCI Insight 2017;2:e90777.

-39 Wilkinson DA, Pandey AS, Thompson BG, Keep RF, Hua Y, Xi G: Injury mechanisms in acute intracerebral hemorrhage. Neuropharmacology 2017;doi: 10.1016/j.neuropharm.2017.09.033.

-40 Zhang Z, Zhang Z, Lu H, Yang Q, Wu H, Wang J: Microglial polarization and inflammatory mediators after intracerebral hemorrhage. Mol Neurobiol 2017;54:1874-1886.

-41 Simon DW, Aneja RK, Alexander H, Bell MJ, Bayir H, Kochanek PM, Clark R: Minocycline Attenuates High Mobility Group Box 1 Translocation, Microglial Activation, and Thalamic Neurodegeneration after Traumatic Brain Injury in Postnatal Day 17 Rats. J Neurotrauma 2017:130-138.

42 Zhu HT, Bian C, Yuan JC, Liao XJ, Liu W, Zhu G, Feng H, Lin JK: Hyperbaric oxygen therapy ameliorates acute brain injury after porcine intracerebral hemorrhage at high altitude. Crit Care 2015;19:255.

43 Kate MP, Choi V, Mouridsen K, Hansen MB, Ostergaard L, Jespersen S, Hill MD, Demchuk AM, Coutts SB, Butcher KS: Blood pressure reduction does not result in perihematoma misery perfusion: A CT perfusion study. Cerebrovasc Dis 2013;353:591.

44 Zaghloul N, Nasim M, Patel H, Codipilly C, Marambaud P, Dewey S, Schiffer WK, Ahmed M: Overexpression of extracellular superoxide dismutase has a protective role against hyperoxia-induced brain injury in neonatal mice. Febs J 2012;279:871-881.

-45 Lan X, Han X, Li Q, Wang J: (-)-Epicatechin, a Natural Flavonoid Compound, Protects Astrocytes Against Hemoglobin Toxicity via Nrf2 and AP-1 Signaling Pathways. Mol Neurobiol 2017;54:7898-7907. 


\section{Cellular Physiology Cell Physiol Biochem 2018;46:2173-2186 \begin{tabular}{l|l|l} 
DOI: 10.1159/000489547 & $\begin{array}{l}\text { O 2018 The Author(s). Published by S. Karger AG, Basel } \\
\text { www.karger.com/cpb }\end{array}$
\end{tabular}}

46 Hazelton JL, Balan I, Elmer GI, Kristian T, Rosenthal RE, Krause G, Sanderson TH, Fiskum G: Hyperoxic reperfusion after global cerebral ischemia promotes inflammation and long-term hippocampal neuronal death. J Neurotrauma 2010;27:753-762.

47 Proctor JL, Scutella D, Pan Y, Vaughan J, Rosenthal RE, Puche A, Fiskum G: Hyperoxic resuscitation improves survival but worsens neurologic outcome in a rat polytrauma model of traumatic brain injury plus hemorrhagic shock. J Trauma Acute Care 2015;79:S101-S109.

-48 Rincon F, Kang J, Maltenfort M, Vibbert M, Urtecho J, Athar MK, Jallo J, Pineda CC, Tzeng D, McBride W, Bell R: Association between hyperoxia and mortality after stroke. Crit Care Med 2014;42:387-396.

-49 Jeon SB, Choi HA, Badjatia N, Schmidt JM, Lantigua H, Claassen J, Connolly ES, Mayer SA, Lee K: Hyperoxia may be related to delayed cerebral ischemia and poor outcome after subarachnoid haemorrhage. J Neurol Neurosurg Psychiatry 2014;85:1301-1307.

-50 Yang S, Chen Y, Deng X, Jiang W, Li B, Fu Z, Du M, Ding R: Hemoglobin-Induced nitric oxide synthase overexpression and nitric oxide production contribute to blood-brain barrier disruption in the rat. J Mol Neurosci 2013;51:352-363.

-51 Yang J, Li Q, Wang Z, Qi C, Han X, Lan X, Wan J, Wang W, Zhao X, Hou Z, Gao C, Carhuapoma JR, Mori S, Zhang J, Wang J: Multimodality MRI assessment of grey and white matter injury and blood-brain barrier disruption after intracerebral haemorrhage in mice. Sci Rep 2017;7:40358.

52 Gobbel GT, Chan TY, Chan PH: Nitric oxide- and superoxide-mediated toxicity in cerebral endothelial cells. J Pharmacol Exp Ther 1997;282:1600-1607.

53 Chen J, Zeng YY, Zeng HL, Chen XY, Su ZX, Liu JW, Wang QF, Li W, Wu WY: Inhibitory effects of pretreatment with Buyanghuanwu decoction on inflammatory cytokine expressions in the kidneys of rats after induction of brain death. J Chin Med Mater 2009;32:1855-1860.

-54 Liu Y, Lin R, Shi X, Fang Z, Wang W, Lin Q, Zhang J, Zhang H, Ji Q: The roles of buyang huanwu decoction in anti-inflammation, antioxidation and regulation of lipid metabolism in rats with myocardial ischemia. Evid Based Complement Alternat Med 2011;2011:561396.

55 Yang S, Gao Q Xing S, Feng X, Peng L, Dong H, Bao L, Zhang J, Hu Y, Li G, Song T, Li Z, Sun J: Neuroprotective effects of Buyang Huanwu decoction against hydrogen peroxide induced oxidative injury in Schwann cells. J Ethnopharmacol 2011;137:1095-1101.

56 Shen J, Zhu Y, Huang K, Jiang H, Shi C, Xiong X, Zhan R, Pan J: Buyang Huanwu Decoction attenuates H2O2induced apoptosis by inhibiting reactive oxygen species-mediated mitochondrial dysfunction pathway in human umbilical vein endothelial cells. BMC Complement Altern Med 2016;16:154.

57 Liao CL, Tong L, Chen YY: Effect of Buyanghuanwu decoction on neuronal nitric oxide synthase expression after permanent focal cerebral ischemia in rats. Acad J First Med Coll PLA 2004;24:864-891.

58 Yang G, Fang Z, Liu Y, Zhang H, Shi X, Ji Q Lin Q Lin R: Protective effects of chinese traditional medicine buyang huanwu decoction on myocardial injury. Evid Based Complement Alternat Med 2011;2011:930324.

-59 Sutherland C, Tebbey PW, Granner DK: Oxidative and chemical stress mimic insulin by selectively inhibiting the expression of phosphoenolpyruvate carboxykinase in hepatoma cells. Diabetes 1997;46:17-22.

-60 Su X, Wang H, Zhu L, Zhao J, Pan H, Ji X: Ethyl pyruvate ameliorates intracerebral hemorrhage-induced brain injury through anti-cell death and anti-inflammatory mechanisms. Neuroscience 2013;245:99-108. 